\section{A computer program to define types by configural frequency analysis}

\author{
JOHN D. MORRIS \\ Georgia Southern College, Statesboro, Georgia 30458
}

Lienert and Krauth (1975) have described a nonparametric method. Configural Frequency Analysis (CFA), through which the existence of modal types of subjects may be tested based on the subjects protiles on dichotomous variable scores. This technique has potential in areas ranging from clinical diagnosis to defining types of students who fail educationally.

The rationale for CFA is to compare the number of occurrences of a specitied profile on the dichotomous variables with the frequency which would be expected from the independent marginal variable frequencies. The expected frequencies are calculated from the observed frequencies of subjects falling into all possible combinations of the values of the dichotomous variables. Each type so specitied is then tested for significance through approximations to the binomial model. With $\mathrm{p}$ dichotomous variables, there are $2 \mathrm{p}$ possible types which could be tested. In order to maintain a desired experimental error rate, each type is tested at an error rate level derived by dividing the input experimental error rate by the number of types for which a test is desired.

Description. The program written to accomplish the CFA analyzes problems with up to 10 dichotomous variables (1,024 types). Hierarchal CFA can also be accomplished at the user's option. Hierarchal CFA merely goes through a separate CFA with all subsets of dichotomous variables to find the most useful subset in defining modal types.

Input. The user enters a variable format for reading subject profiles, the number of subjects, number of variables, number of types to be tested, over-all experimental error rate desired, input unit number, the alphameric character signifying the positive instance of each variable. and the a priori chosen types for which a test is desired (alternatively zero types to be tested can be specified and all $2 \mathrm{p}$ will be tested).

Output. The program output includes the observed and expected frequencies, the approximate associated binomial probability level estimated both by the normal and Camp-Paulson methods as suggested by Lienert and Krauth (1975) and a test of significance for each a priori specified type with the adjusted error rate. If the hierarchal option is specified, the subset of variables giving the most clear-cut types are reported.

Computer and language. This FORTRAN IV program has been run on both the IBM $370 / 165$ and CDC CYBER70 and has been used in both interactive and batch modes.

Availability. The program with complete documentation, sample input. and output is available at no cost on request from the author at: Department of School Services, Box 8143, Georgia Southern College. Statesboro. Georgia 30458.

\section{REFERENCE}

Lienert, G. A., \& Krauth. J. Configural frequency analysis as a statistical tool for defining types. Educational and Psychological Measurement, 1975. 35. 231-238. 